\title{
INFORMATIONSDIENST
}

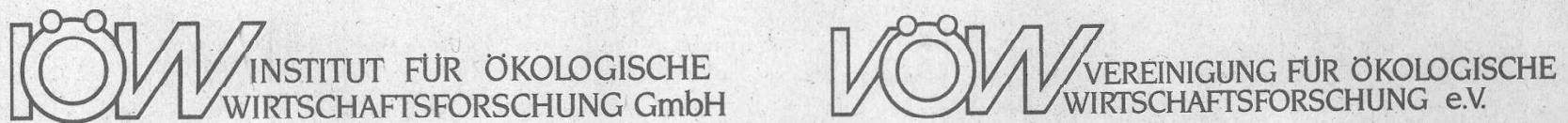

\section{Lernen zu lernen zu lernen ...}

Können nicht nur Individuen, sondern auch ganze Organisationen lernen? Die Seriosität dieser Frage ist durchaus umstritten, $d$. h. daß Unternehmen als lernfähige (und lernende) soziale Systeme beschrieben werden können, ist keineswegs allgemein anerkannt. In der editorischen Einleitung einer Managementzeitschrift (Harvard Business manager 1/94) heißt es kategorisch: "So sind streng genommen Organisationen als Ganzes zum Lernen gar nicht imstande." Zwar hat seit Heinen und damit seit Ende der 60er Jahre die Betriebswirtschaftslehre damit begonnen, sich sozialwissenschaftlich zu öffnen. Unter dem Druck einer kulturell auf einen verengt-kalkulatorischen Begriff des Ökonomischen festgelegten Praxis entwickelten sich freilich kaum wegweisende organisationstheoretische Reflexionen, sondern es gewann eine Managementlehre die Oberhand, die sich allzuhäufig in technokratisch-euphorischer Machermentalität von zahlreichen Unternehmensberatungen kaum unterscheidet.

Am sich intensivierenden organisationstheoretischen Diskurs ist bislang nur eine verschwindend geringe Zahl akademischer Betriebswirte beteiligt. Eben darum fanden wir es reizvoll, den thematischen Schwerpunkt dieses Informationsdienstes (Seite 1 bis 18) ausschließlich von Vertretern des Faches Betriebswirtschaftslehre gestalten zu lassen. Die Frage nach der ökologischen Lernfähigkeit halten wir für einen zentralen Erklärungsansatz, um die ökologischen Handlungspotentiale und Handlungsrestriktionen von Unternehmen aufzuzeigen. Wir hoffen, mit dem folgenden eine interessante Zusammenstellung für den betriebswirtschafts-theoretischen und ökologischen Diskurs vorlegen zu können. (Klaus Fichter/Reinhard Pfriem)

Aus der »klassischen « betriebswirtschaftlichen Perspektive heraus, wie sie maßgeblich von Gutenberg begründet wurde, als main-stream von Allgemeiner Betriebswirtschaftslehre aber auch 1994 noch die Studierenden belastet, ist die Konzentration auf organisationstheoretische Fragestellungen nicht weniger als die Existenzvernichtung ihrer Ausgangsannahme: denn Gutenberg versuchte nicht weniger, als das Fach auf (neoklassisch-mikroökonomisch gedachte) »betriebswirtschaftliche Grundvorgänge engzuführen und damit »die Unternehmung als Gegenstand betriebswirtschaftlicher Theorie in eine Ebene zu projizieren, in der zwar Organisation vorhanden ist, aber nur eine solche, die der eigenen Problematik entbehrt. « (Gutenberg 1929, S. 25 f.) Und eine Ökonomie, die Geld, oder eine Soziologie, die Macht als Steuerungs- medium verabsolutiert, haben sich von den Problemen organisationalen Lernens tunlichst freizuhalten: die Frage nach den Bedingungen der Möglichkeit ökologischer Unternehmenspolitik schrumpft hier auf jene nach hinreichenden finanziellen Anreizen oder genügendem politischen Druck zusammen.

Gegenüber diesen beiden Abteilungen allzu schlichter. Weltbilder scheinen differenzierte organisationstheoretische Untersuchungen mittlerweile erfreulich im Vormarsch zu sein (wenn auch noch kaum in der Betriebswirtschaftslehre). Wegbereitend für die Öffnung zu wirklichkeitstüchtigen Theorien war die Einsicht in »bounded rationality « (Simon 1957), d. h. die Klarstellung, daß auch bei bestem Willen Entscheidungsträger nur arg begrenzt rational handeln können. In unseren eigenen Praxisforschungs-

\section{Aus dem Inhalt}

3 IÖW-Jahrestagung Ökologie und Innovation Blockaden, Bedingungen, Ziele

4 Ökologische Lernfähigkeit: Maßstab für die Qualität der Unternehmensentwicklung Sabine Harde

9 Unternehmen als Lernende Systeme Peter Wengelowski, Thomas Breisig

11 Ökologische Lern- und Entwicklungsprozesse von Unternehmen Thomas Dyllick, Frank Belz

12 Kriterien für die Umwelt-Lernfähigkeit von Unternehmen Anja Grothe-Senf

14 Organisationales Lernen und betrieblicher Umweltschutz

Hans-Gerd Ridder

16 Umweltkommunikation als wechselseitiges Lernen zwischen Unternehmen und Anspruchsgruppen Klaus Fichter

18 Die Lernfähigkeit von Unternehmen am Beispiel des ökologischen Umsteuerns in der Automobilindustrie Hartmut Kreikebaum

19 Die Karawane zieht weiter. Zur umweltpolitischen Relevanz der Umweltökonomie Gerhard Maier-Rigaud

20 The roaring fifties das Abheben der Stoffströme Ulrich Petschow

22 Ökologisch verträgliche Mobilität in Stadtregionen Markus Hesse, Rudolf Petersen

25 Eine gesamtökologische Betrachtung von Produktstammbäumen Bausteine für ein Stoffstrommanagement Engelbert Schramm, Stefan Zundel

27 Kompetenzwerkstatt Bergisches Städtedreieck Rainer Lucas

28 Privatisierung der Sanierungsgesellschaften im Lausitzer Braunkohlerevier Berthold Minthe

29 VÖW-Tagungsbericht 
projekten hat sich immer wieder bestätigt, was wir selbst als doppelte Subjektivität informatorischer Prozesse bezeichnen:

(1) stützt sich aus Gründen des Entscheidungsdrucks jede Entscheidung nur auf eine Teilmenge der Informationen, die »eigentlich « nötig wären;

(2) unterliegt die Verarbeitung der eingeholten Informationen höchst subjektiven (systemspezifischen oder individuellen) Selektionskriterien.

Daher ist die Vorstellung gleichförmiger Anpassungsprozesse obsolet (wie sie eine ökonomistische und immer noch an eindeutig-optimale Entscheidungen glaubende Betriebswirtschaftslehre nahelegt).

Auf die scheinbar selbe Information reagieren Systeme unterschiedlich: die einen gründen als Lobby für möglichst lang anhaltende Produktion von PVC eine PR-Arbeitsgemeinschaft mit dem irreführenden Titel »PVC und Umwelt«, die anderen orientieren - wie zwei große deutsche Chemieunternehmen - auf den Ausstieg aus PVC oder - wie ein Tapetenhersteller - auf die Kennzeichnung des Befundes, daß die Nachfrage nach PVC-Strukturtapeten weiterhin groß ist, als eigenes Problem.

Die Herausbildung des managementtheoretischen Diskurses über strategische Unternehmensführung unter den Schlüsselbegriffen von gesteigerter Komplexität und Unsicherheit läßt sich insofern als Abkehr von frühmodernen Auffassungen über Objektivität und Rationalität oder als Aufstieg zur Einsicht in die Subjektabhängigkeit von Realitätskonstruktionen deuten. Instrumentell richtete sich sich der Blick im Rahmen strategischer Frühwarnsysteme (FWS) zunehmend auf »weak signals« (schwache Signale, Ansoff), und in der weiteren Entwicklung wurde versucht, strategische Frühwarnsysteme $\mathrm{zu}$ strategischen Früherkennungssystemen (FES) zu transformieren. Der dabei zugrundeliegende geistige Fortschritt hatte und hat, stillschweigend zwar, paradigmatische Charakterzüge:

(1) An die Stelle von (zeitlich kurzfristigen und methodisch ohne weiteres quantifizierbaren) Erfolgen treten (zeitlich langfristige und methodisch in erster Linie nur qualitativ beschreibbare) Erfolgspotentiale.

(2) Bezogen auf den throughput (stofflich die Verwandlung von Input in Output, finanzökonomisch der Prozeß G-W-G') verändert sich das Kristallisationsfeld: die Dominanz von Rationalisierung und Kostensenkung wird abgelöst durch den Vorrang von zukünftigen Marktchancen und (wiederum) Erfolgspotentialen. Die wachsende Beschäftigung mit den Möglichkeiten, die Szenarienmethode für strategische Unternehmensführung anzuwenden (pionierhaft: von Reibnitz 1987) indiziert u.a. die Blickwende vom Input zum Output.

\section{Abkehr von Modellen reiner Gewinmaximierung}

Strategie als Suchprozeß einer Unternehmung verstehen zu lernen, bedeutet jedenfalls die Abkehr von allen (der Tendenz nach objektivistischen und rationalistischen) deduktiv-logischen Modellen »reiner « Gewinnmaximierung, einschließlich solcher trivialer Denkfiguren wie der Erfahrungskurve, nach der Unternehmen in einer wachsenden Branche im Vergleich zur Konkurrenz ihre Produktion rascher ausweiten, $\mathrm{zu}$ niedrigeren Preisen anbieten und so neue Marktanteile erobern sollten.

Unternehmen stehen sich als Organisationen (bei uns synonym: soziale Systeme) bei der Bewältigung ökologischer Probleme häufig selbst im Wege. Ausnahmslos jeder, der aus den letzten 10 Jahren heraus sich das Abzeichen des ökologischen Pionierunternehmers anheften möchte (bzw. legitimerweise kann), hält bei möglichst jeder Gelegenheit Reden darüber, daß sich der Staat heraushalten möge. Gleichzeitig werden in der bislang repräsentativsten Unternehmensbefragung (s. FUUF 1991) gesetzgeberische Initiativen als wichtigster Anreiz zu betrieblicher Umweltpolitik ermittelt. Dieser beschränkten (präziser: widersprüchlichen) Sicht nach außen entspricht eine ebenso beschränkte (und widersprüchliche) Sicht nach innen: wenn ökologische Unternehmenspolitik trotz der Formel »Umweltschutz ist Chefsache« nämlich primär als (delegiertes) Ressort behandelt wird oder die Sache individueller Promotoren auf Geschäftsführungsebene bleibt, die sich in der Organisation nicht durchsetzen und/oder in ihrer Stellung nicht halten können.

Demgegenüber kann definiert werden: $\gg$ Lernende Organisationen sind in fünf Hauptverrichtungen besonders tïchtig; systematischem Problemlösen, Experimentieren mit neuen Ansätzen, Lernen aus eigenen Erfahrungèn, Lernen aus den Erfahrungen und erfolgreichen Praktiken anderer sowie raschem und effektivem Übertragen von Wissen in der ganzen Organisation. « (Garvin 1994, S. 76)

\section{Entscheidung mittels Selektion}

Unternehmen sind keine Trivialmaschinen. Ihren Selbstorganisationsprozessen (vgl. dazu Probst 1987) ist Bedeutung beizumessen. Unternehmen konstruieren ihre Realität selbst (akademische Betriebswirte können an diesen Realitätskonstruktionen nach eigenem gusto mehr oder weniger teilnehmen). Unternehmenspolitische Entscheidungen sind insofern nicht durch die Umwelt(en) determiniert, sondern kommen zustande über die Selektionen, die ein Unternehmen vornimmt. Solche Selektionen sind allenfalls untergeordnet rechenhaft: sie speisen sich aus Traditionsbezügen und gewachsenen Werten, sie oszillieren um kreative Zukunftsvorstellungen und die dementsprechende Gestaltung des Kommunikationsnetzes.
Dabei kommen (1) das Werte-Prinzip und (2) das Interdependenz-Prinzip von Freeman/Gilbert zur Geltung: »Das Handeln von Individuen und Organisationen ist teilweise durch die Werte dieser Individuen und Organisationen bestimmt ... Der Erfolg einer Organisation beruht teilweise auf den Entscheidungen und Handlungen der mit der Organisation verbundenen Interessengruppen«. (Freeman/Gilbert S. 23) Organisationales Lernen hat demzufolge (a) mit Sinn und (b) mit Kooperation zu tun. Sinn muß kommuniziert werden, um relevant zu werden. Hier hat übrigens der seriöse wirtschaftsethische Diskurs seinen Platz: ihm geht es nicht um die Maxime, daß künftig jenseits von Ökonomie allgemein wirtschaftsethisch gehandelt wird, wohl aber um Anstöße, (kritisch) Defiziten ethischer Sinnhaftigkeit stärker nachzugehen und (konstruktiv) die betriebswirtschaftlichen $\mathrm{Be}$ dingungen der Möglichkeiten zu ethisch-sinnhaftem Handeln stärker auszuloten.

In der Interaktion von Wirtschaft und Gesellschaft (vgl. dazu Wiesenthal 1993, S. 8 ff.) kann die Kommunikation von Sinn nur funktionieren, wenn sie anschlußfähig ist, d.h. für gesellschaftliche Anforderungen an Unternehmen: Unternehmen müssen diese Anforderungen in ihrem systemspezifischen Code verarbeiten können. Das u. a. von R.E. Freeman, Peter Ulrich und Thomas Dyllick vertretene Stakeholderoder Anspruchsgruppenkonzept (wonach der strategische Erfolg einer Unternehmung auch von ihren Beziehungen zu nichtmarktlichen Akteuren abhängig ist - zum Anspruchsgruppenmanagement s. ausführlich Janisch 1993) greift dann zu kurz, wenn suggeriert wird, das System Unternehmung könne sich gleichsam qua Willensentscheidung allen internen und externen Anspruchsgruppen gegenüber aufgeschlossen zeigen. Auch die regulative Idee des Konsensus-Managements scheint eine normative Überfrachtung tatsächlicher Interaktionsprozesse zwischen Unternehmen und stakeholders zu sein: realistischer läßt sich eine solche Kommunikation als Verständigungsmanagement beschreiben, mit dessen Hilfe im Einzelfall zwar auch Konsense möglich sind, wonach vor allèm aber mit Differenzen reflektierter umgegangen werden kann. So ergibt sich das Konzept einer bewußt pluralistischen Unternehmenskultur: Pflege der Vielfalt, von Experimenten, des Irrenkönnens und gerade dadurch Kultivieren der Lernfähigkeit. Es lohnt sich, der Hypothese nachzugehen, daß die strategische Überlebensfähigkeit und erst recht die Lernund Fortschrittsfähigkeit einer Unternehmung (s. dazu ausführlich Kirsch) abhängig ist von der selbsterzeugten Pluralität. (Der Vorstand eines Automobilkonzerns, der führende Mitarbeiter entläßt, die über das Auto hínausdenken, schränkt seine strategischen Optionen unnötig ein.)

Bedingungen für die Möglichkeit einer pluralistischen Unternehmenskultur zu analysieren, sprengt selbstverständlich den gedanklichen Rahmen einer rein monetären Kodierung von 
Unternehmenspolitik. Während sich eine wachsende Zahl von Betriebswirtschafts- und Managementwissenschaftlern vor dem dazu zwingenden empirischen Hintergrund mit weichen Daten und strategischen Erfolgspotentialen beschäftigt, verharren anscheinend einige systemtheoretisch sehr aufgeklärte Soziologen zu sehr im Banne ihres großen Meisters. Infolgedessen wird übersehen, daß die Selbstbeobachtung und Selbstbeschreibung von Unternehmen dazu führen kann, den eigenen Code zu erweitern. (Eben dies ist in einem tiefer verstandenen Sinn Entwicklung von Unternehmenskultur.) Organisationsziele werden von Menschen gemacht, von Organisationsmitgliedern geprägt bzw. beeinflußt. Dieser Befund wird durch die Einsicht, daß Unternehmen als soziale Systeme agieren, nicht vom Tisch gewischt und legt der kritischen Unternehmensforschung auf, ihre Handlungsund Akteursorientierung nicht zu vergessen. Praxisforschung achtet auf ihren empirischen Gehalt und versucht, sich gegen allzu selbstbezügliches Theoretisieren zu wappnen.

\section{Hendric Hallay, Reinhard Pfriem} Oldenburg

\section{Literatur}

- Freeman, R.E./Gilbert, D.L. (1991): Unternehmensstrategie, Ethik und persönliche Verantwortung, Frankfurt a. M./New York.

- FUUF/Forschungsgruppe Umweltorientierte Unternehmensführung (Hg.) (1991): Umweltorientierte Unternehmensführung. Möglichkeiten zur Kostensenkung und Erlössteigerung - Modellvorhaben und Kongreß, Reihe Berichte des Umweltbundesamtes, Bd. 11/91, Berlin.

- Garvin, D.A. (1994): Nicht schöne Worte Taten zählen, in: Harvard Business manager $1 / 94$, S. 74-85, Hamburg.

- Gutenberg, E. (1929): Die Unternehmung als Gegenstand betriebswirtschaftlicher Theorie, Berlin.

- Janisch, M. (1993): Das strategische Anspruchsgruppenmanagement. Vom Shareholder Value zum Stakeholder Value, Bern/ Stuttgart/Wien.

- Kirsch, W. (1990): Unternehmenspolitik und strategische Unternehmensführung, München.

- Probst, G.J.B. (1987): Selbstorganisation. Ordnungsprozesse in sozialen Systemen aus ganzheitlicher Sicht, München.

- von Reibnitz, U. (1987): Szenarien - Optionen für die Zukunft, Hamburg.

- Simon, H. (1957): Models of Man. Social and Rational, New York.

- Wiesenthal, H. (1993): Lernchancen der Risikogesellschaft. Über gesellschaftliche Innovationspotentiale und die Grenzen der Risikosoziologie, Berlin.

\section{IÖW-Jahrestagung am 25. und 26. November in Hamburg Ökologie und Innovation - Blockaden, Bedingungen, Ziele}

Die Jahrestagung findet vor dem Hintergrund der sogenannten »Standortdebatte « statt, in der die umweltpolitischen Herausforderungen vorwiegend als Kostenfaktor und Innovationshindernis im Gefolge von ordnungspolitischer Regulierung gesehen werden.

In den Wirtschaftswissenschaften besteht dagegen weitgehend Einigkeit darüber, daß reine Strategien zur Lohnkostensenkung für eine fortgeschrittene Industriegesellschaft keine ernstzunehmende wirtschaftspolitische Alternative sind. Bei der Verschlankung der Produktion, der Reduktion der Lohn- und Lohnnebenkosten muß darauf geachtet werden, da $B$ solche Maßnahmen nicht die Strategiefähigkeit der Unternehmen untergraben: und hier vor allem nicht die technische und soziale Innovationsfähigkeit von Unternehmen.

Auf der anderen Seite sieht die Umweltbewegung traditionell mit dem technischen Fortschritt eher Folgeprobleme verbunden: Atomkraft, Chemisierung der Gesellschaft oder jüngst die Gentechnologie stehen für technologische Entwicklungslinien, die Stein des Anstoßes geworden sind. Und vielfach war die Reaktion von der Idee gespeist, Innovationsprozesse $\mathrm{zu}$ verlangsamen, um sie einer gesellschaftlichen Kontrolle unterwerfen zu können. Andererseits kann sich auch die Umweltbewegung der sozialen und technischen Entwicklungsdynamik einer modernen Industriegesellschaft nicht entziehen. Die Auseinandersetzung verlagert sich daher mehr und mehr auf die Art der Innovationen, auf ihre $\mathrm{Na}$ turgemäßheit, Nachhaltigkeit bzw. auf die jeweiligen Risikopotentiale der konkret angestrebten stofflich-technischen Entwicklungspfade, sei es nun im Energie- und Verkehrsbereich oder in der Chemie und Biotechnologie. Die Forderung nach integriertem Umweltschutz ist ein weiteres Motiv, sich aktiv mit dem Thema ökologischer Innovationen auseinanderzusetzen.

Innovation wird somit zu einem Schlüsselbegriff, sowohl im wirtschaftspolitischen als auch im ökologischen Diskurs. Und der Gedanke ist naheliegend, gleichsam zwei Probleme, die strukturelle ökonomische Krise und die ökologische Herausforderung der modernen Industriegesellschaft mit einer Strategie der ökologischen Innovation anzugehen. Was aus politischer Sicht naheliegend ist, muß allerdings aus wissenschaftlicher Perspektive nicht zwingend sein. Die Blockaden, Bedingungen und Ziele, kurz: das Erfolgspotential einer solchen Strategie ist Gegenstand der Tagung.
Vor diesem Hintergrund befaßt sich die Tagung mit folgenden Themen:

- Traditionell ist der technische Fortschritt ein »schwieriges « Thema in den Wirtschaftswissenschaften. Es paßt nicht so recht in den neoklassischen Mainstream. Theoretische Entwürfe, die sich dezidiert mit Innovatio-

nen auseinandersetzen, beispielsweise solche schumpeterianischer Provenienz, werden weitaus weniger rezipiert. Wir fragen deshalb: Auf.welche theoretischen Konzepte kann sich die Strategie der ökologischen Innovation stützen ?

- Innovationen lassen sich nicht verordnen, und ob der großzügige Einsatz von staatlichen Fördermitteln auf diesem Gebiet auch das gewünschte Resultat zeitigt, ist zumindest eine offene Frage. Die Bedingungen, unter denen es zu Innovationen kommt, sind komplex. Wir fragen deshalb: Was sind die Voraussetzung für Innovationsfähigkeit im allgemeinen und was die einer ökologischen Innovationsfähigkeit im besonderen?

- Ökologische Kriterien spielen schon heute bei dem Design von neuen Produkten und der Implementation neuer Produktionsprozesse, aber auch bei strukturellen Veränderungen des Wirtschaftsgefüges eine wichtige Rolle. Wir fragen deshalb: Welche Felder und Akteursstrukturen sind als Felder für eine ökologische Innovationsstrategie erkennbar und von Relevanz?

- Über die konkrete Ausformung von Technikpfaden wird zunehmend in >Innovationssystemen auf nationaler und standortbezogen auf regionaler Ebenen mit dem Ziel der Formung von Innovationsbündnissen gestritten und verhandelt. Neben staatlichen Institutionen und den Tarifpartnern spielen in diesen Innovationssystemen die wissenschaftliche Infrastruktur und zahlreiche >zivilgesellschaftliche $<$ Akteure eine wichtige Rolle. Berufsverbände (z. B. VDI), halbstaatliche Institutionen (z.B. TÜV, Normungsausschüsse wie DIN), die Medien und natürlich auch die neuen sozialen Bewegungen zählen dazu. Wir fragen deshalb: Welches Rollenverständnis erfordert eine Strategie der ökologischen Innovation von den beteiligten Akteuren, welcher institutioneller Arrangements bedarf es für die $\mathrm{Si}$ cherung einer nachhaltigen Lebensweise?

Für nähere Informationen steht Karin Robinet im IÖW-Büro Hamburg zur Verfügung (Tel. 040/ 371294). 
(c) 20I0 Authors; licensee IÖW and oekom verlag. This is an article distributed under the terms of the Creative Commons Attribution Non-Commercial No Derivates License (http://creativecommons.org/licenses/by-nc-nd/3.o/), which permits unrestricted use, distribution, and reproduction in any medium, provided the original work is properly cited. 